\title{
Die heile Welt der Angst
}

\author{
Heinz-Michael Just
}

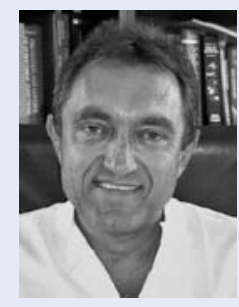

Heinz-Michael Just
„Die Angst vor Keimen ist für die Industrie ein Milliardengeschäft" titelte eine Sendung des NDR im Oktober dieses Jahres. Und ein Blogger fragt, bezogen auf antibiotikaresistente Keime: „Wie kann es sein, dass eine normalerweise so zur Furcht bereite Gesellschaft wie unsere, so völlig gleichgültig ihrer größten Bedrohung seit dem Schwarzen Tod entgegen driftet?“ (http:// www.scilogs.de/fischblog/warum-habt-ihr-keineangst/)

Das eine bedingt das andere: ohne Angst kein Milliardengeschäft, oder besser: wegen der Angst ein Milliardengeschäft. Die unsichtbare Gefahr, die angeblich überall auf uns lauert: in der Luft (die Keime im OP), im Wasser (voller Legionellen und Pseudomonaden) und auch sonst überall. Deshalb brauchen wir von Türgriffen und Lichtschaltern aus Kupfer, über antimikrobiell beschichtete Oberflächen, Vorhänge und Kacheln für Fußboden und Wände, antimikrobiell imprägnierte Bausteine und selbstdesinfizierende Siphons bzw. Desinfektionsgeräte für die ach so kritischen Geruchsverschlüsse bis hin zu „Luftwäschern“ und imprägnierter Wäsche, - alternativ mit Silberfäden durchwoben - ein antimikrobielles Rundum-Sorglos-Paket für Klinik, Praxis und Zuhause. So suggeriert es uns jedenfalls die Industrie - aus Angst um unsere Gesundheit natürlich, ganz unaufgeregt. Und wie das verfängt, zeigt die aufgeregte, fast schon hysterische Angst des Bloggers.

Wir brauchen aber etwas ganz anderes und letztlich viel mehr! Wir brauchen zum einen Wissen um die Herkunft und Verbreitung von Mikroorganismen und zum anderen epidemiologische Erkenntnisse, unter welchen Voraussetzungen es zu Infektionen kommt. Das erste muss nämlich nicht automatisch zum zweiten führen! Das Infektionsschutzgesetz verpflichtet uns, „die nach dem Stand der medizinischen Wissenschaft erforderlichen Maßnahmen“ durchzuführen, um „die Weiterverbreitung von Krankheitserregern, insbesondere solcher mit Resistenzen, zu vermeiden“- unabhängig von der Gefährdung für eine Infektion. „Insbesondere“ heißt aber, nicht nur, sondern auch: also alle „Krankheitserreger“, unabhängig von der Resistenz. Wenn man sich jetzt vor Augen hält, dass Staphylococcus epidermidis (unser primärer „Hautschutz“-Keim) als der häufigste Erreger der Gefäßkatheter-assoziier- ten Sepsis gehandelt wird, dann beginnt man zu ahnen, dass hier etwas nicht stimmen kann; insbesondere, wenn eine Klinik in Deutschland (http://www.derwesten.de/staedte/bochum/patienten-moegen-gruss-verbot-von-bochumer-klinikaerzten-id9728110.html) als Konsequenz ein Händeschüttel-Verbot für ihre Beschäftigten erlassen hat - entgegen jeder infektiologischen und erst recht medizinischen Vernunft!

Angst basiert (meist) auf Unkenntnis, aber auch auf „Kenntnis“: Aus Unkenntnis natürlicher und infektiologischer Zusammenhänge (s. Händeschüttel-Verbot) oder aus Kenntnis, d.h. dem Bewusstsein der eigenen Verantwortung z.B. bei Mitarbeitern von Aufsichtsbehörden. Diese Angst vor der eigenen Verantwortung kann dann dazu führen, dass hartnäckig auf regelmäßige Desinfektion von Flächen gepocht wird (Begründung: der Handkontakt), ordnerweise „Hygienepläne“ kontrolliert werden (dann sieht man schwarz auf weiß, dass alles gut ist) oder die räumliche Isolierung bei MRSA-Nachweis ohne wenn und aber gefordert wird. Schematisches Abarbeiten von Checklisten beruhigt und schafft die Illusion von Sicherheit.

Wer einmal in Krankenhäusern anderer (auch europäischer) Länder war und wer die Bilder der Behandlung an Ebola Infizierter im Fernsehen bewusst gesehen hat, dem müssten eigentlich Zweifel kommen, ob wir auf angemessenem Niveau diskutieren. Wir haben die besten technischen wie therapeutischen Voraussetzungen - und gleichzeitig die größte Angst. Es gibt wohl wirklich das „Sicherheitsparadox“, was bedeutet, „dass die Empfindlichkeiten für Unsicherheiten mit dem Ausmaß der Sicherheit wächst“" [1].

Trotz sinnvoller Empfehlungen für die Händehygiene achten wir nicht darauf, durch unsere Hände niemanden zu gefährden (sonst müsste man Ärzte nicht immer wieder auf die Händedesinfektion hinweisen, wo sie erforderlich ist), sondern wir trachten danach, dass alles möglichst keimfrei sein sollte, wo wir hinlangen. Die „keimfreie“ Umgebung ist aber ein Synonym für Widernatürlichkeit; trotzdem scheint das unser höchstes Ziel zu sein. Natürlich gibt es Mikroorganismen, mit denen wir natürlicherweise keinen Kontakt haben und die uns krank werden lassen, wenn sie uns besie- 
deln (z. B. Influenza, Ebola), aber wir ängstigen uns vor den eigenen Bakterien, die seit Menschengedenken in friedlicher Symbiose mit uns leben und nur dann zur Gefahr werden können, wenn sie Zugang zu Bereichen unseres Körpers bekommen, wo keine Abwehr vorgesehen ist. Und dass dies - im Sinn von nosokomialen Infektionen - passieren kann, liegt an uns Ärzten, nicht an den Bakterien. Wir durchstechen die Haut, schneiden sie auf, intubieren, legen Katheter oder implantieren Fremdkörper. Hierbei haben wir die Einhaltung der Regeln der Asepsis zu garantieren und nicht, weil wir zu schwach dazu sind, Kupfer, Silber, Nano und anderes zu Hilfe zu rufen. Wenn wir sorgfältig arbeiten und uns korrekt verhalten (würden), bräuchten wir die Aufmerksamkeit nicht von uns wegzulenken. Staphylococcus aureus ist der dominierende Erreger postoperativer Wundinfektionen. In Deutschland rechnet man, dass bis zu 20\% davon MRSA sind. Um diesen Anteil wird ein Riesen-Hype gemacht und über die restlichen 80\% MSSA (vier Fünftel aller Infektionen!) redet niemand. Die ist man ja gewohnt, aber die MethicillinResistenz, die adelt den Keim als Kumpanen des Schwarzen Todes!?

Vielleicht sollten wir uns eher um die vier Fünftel anstatt um das eine Fünftel kümmern. Indem wir nicht eine Eigenschaft eines Bakteriums (die laut Arbeitsschutz kein Gefährdungsmerkmal darstellt! [2]) als das Problem sehen, sondern die Tatsache, dass wir ihm das Eindringen in unseren Körper ermöglichen! Erste Studien zeigen den Weg in die richtige Richtung $[3,4]$. Die bislang so vertrauten Gepflogenheiten halten dagegen einer wissenschaftlich-kritischen Betrachtung nicht stand und bedürfen einer zeitgemäßen Überprüfung in Form evidenzbasierter Studien [5]. Kombiniert mit einem Umdenken inkl. der Frage, ob es nicht besser wäre, einen Patienten, der durch unsere operativen Eingriffe (z. B. künstl. Gelenkprothesen, Herzklappen, Gefäße) gefährdet wird, ab stationärer Aufnahme bis einige Tage nach dem Eingriff so lange zu isolieren, bis er keiner erhöhten Infektionsgefährdung mehr ausgesetzt ist. Dies würde die Sorgfalt des Behandlers demonstrieren und der Patient hätte dafür Verständnis, weil es zu seiner Sicherheit geschieht. Stattdessen kommen wir von unseren eingefahrenen Verhaltensmustern nur schwerlich los [6]. Zwar öffnen wir uns wenngleich sehr zaghaft weil ängstlich - der risikobezogenen Betrachtung, aber letztlich unterliegen wir unserer immer noch übermächtigen Angst [6]; siehe auch S. 226-228 in diesem Heft. Obwohl wir es eigentlich besser wissen [5]. Heinz Bude nennt es die „Erkenntnis, dass wir uns selbst die Angst machen, die wir im Blick auf einen bedrohlichen Zustand ... erleben“.

Die Zunahme der Resistenzen nahezu aller Bakterien gegen Antibiotika wird uns zwingen umzudenken, denn mit unseren angstgesteuerten Verhaltensweisen der Vergangenheit sind wir zum Scheitern verurteilt. Wir müssen uns der Realität stellen und bekommen gezeigt, dass überzogene Sicherheitserwartungen von der Gesellschaft jetzt bereits abgelehnt werden [7].

„Angst zeigt uns, was mit uns los ist“ [1], wir sollten nun endlich unsere Augen aufmachen und ggf. gefärbte Brillen absetzen.

\section{Literatur}

1 Bude H. Gesellschaft der Angst. Hamburger Edition HIS Verlagsges. mbH; 2014

2 TRBA 250. http://www.baua.de/de/Themen-von-A-Z/Biologische-Arbeitsstoffe/TRBA/pdf/TRBA-250.pdf?_blob=publicationFile

3 Huang S. S. et al. Targeted versus Universal Decolonization to Prevent ICU Infection N Engl J Med 2013; 368: 2255 - 2265

4 Climo M. W. et al. Effect of daily chlorhexidine bathing on hospital-acquired infection. N Engl J Med 2013; 368: 533 - 542

5 Fätkenheuer $G$ et al. Screening and isolation to control meticillin-resistant Staphylococcus aureus: sense, nonsense, and evidence. http://www.sciencedirect.com/science/article/pii/ S0140673614606607

6 KRINKO Empfehlungen zur Prävention und Kontrolle von Methicillin-resistenten Staphylococcus aureus-Stämmen (MRSA) in medizinischen und pflegerischen Einrichtungen.

7 Heppekausen C, Heuberger S. Neuere Rechtsprechung zur Haftung bei Hygienemängeln. KHHup2date 2014; 9: 151 156 